\title{
MOBILE GAS CHROMATOGRAPHS COUPLED WITH MASS AND ION MOBILITY SPECTROMETERS AND THEIR APPLICATIONS
}

\begin{abstract}
Chemical analysis of different materials at the place where analytes are present (on-site analysis) has several advantages in comparison to analysis of these materials after delivering the samples to laboratory. Mobile devices, possessing expected properties in terms of using energy, mass and volume are needed for such analyses. The obtained results should be comparable to those obtained with the stationary instruments. Mass and ion mobility spectrometers are examples of the instruments fulfilling these requirements. At the beginning, the article describes the developments in combining of mass and ion mobility spectrometers (MS, IMS) with miniature gas chromatographs (GC). Both systems are used for analyses in the field, mainly for determination of environmental pollutions. They are used not only for analysis of typical chemicals present in different environmental compartments (in air, water and soil samples) but also for analysis of explosives, drugs and chemical warfare agents when fast results are needed. Particularly noteworthy is their applications in space exploration on the International Space Station. The selected examples of applications of miniaturised GC-MS and GC-IMS devices are presented in the second part of this mini review.
\end{abstract}

Keywords: gas chromatography, mass spectrometers, ion mobility spectrometers, miniaturisation, mobile instruments

\section{Introduction}

The contemporary trend in many fields of analytical chemistry is to miniaturise measuring devices. It is related with the observed movement to perform analysis at the place where analytes are present [1]. The miniaturisation of conventional analytical instruments can provide many attractive features, e.g. better flexibility and faster results, without sacrifice in performance, and perhaps, with improved performance. A possibility of performing on-site analysis is becoming more important, especially in environmental applications (air, water and soil pollutants). Such approach reduces risk of contamination, sample loss and sample decomposition during transport. Increasingly, there is strong need to get reliable results of analysis with shorter turnaround times. It is especially important in military, strengthening security, forensic and antiterrorist operations, in the areas of food safety and for determination of pharmaceutical contaminants.

\footnotetext{
${ }^{1}$ Institute of Chemistry, Military University of Technology, ul. gen. S. Kaliskiego 2, 00-908 Warszawa 46, Poland, email: witkiew@wp.pl

${ }^{2}$ Department of Analytical Chemistry, Chemical Faculty, Gdańsk University of Technology, ul. G. Narutowicza 11/12,80-233 Gdańsk, Poland, email: waldemar.wardencki@pg.edu.pl

*Corresponding author: waldemar.wardencki@pg.edu.pl
} 
The miniaturisation of the complex separation systems is one of the directions of development of separation system. Today mobile, transportable and portable, gas chromatographs [2] are universally used for determination of environmental pollution, including so specific compounds, as chemical warfare agents (CWA). They are also applied in industrial applications, especially for analyses in the field. Some portable GC systems can work unattended for a long periods of time as monitoring devices sending signals, e.g. from the ocean or from the space to the Earth.

The performance of conventional and miniaturised GC systems rely heavily on the detector. Nearly all types of the detectors used in laboratory instruments may be used in mobile chromatographs but their properties should match the other components of micro chromatographs (micro GC).

The detectors for miniature GC need to be small in size and fitted to the small flow within the microfluidic channels. The advances in micro technology, mainly in microelectromechanical (MEMS) technology, in combination with microfluidic systems, enable to miniaturise conventional gas chromatography detectors, or even, to develop the novel detectors based on new principles for micro GC. Detectors for micro GC are still developed in terms of miniaturisation, sensitivity and mass production. The further miniaturisation is possible by using nanoelectromechanical (NEMS) technology. Recently, an interesting review on miniaturisation of conventional GC detectors and the developments of novel detectors for micro GC chromatography has been published [3].

Mass spectrometers are very useful for miniaturisation of micro gas chromatographs. Mass spectrometers (MSs) are considered to be one of the most desirable detectors for chromatography. Therefore, mass spectrometers are good candidates for micro GC because miniaturised columns reduce vacuum pump requirements, improve separation efficiency, accommodate very small samples (especially important in biomedical applications) and improve detection limits. In turn, ion mobility spectrometers (IMSs), due to their principle of operation and low energy consumption, may be relatively easy combined with portable GC.

\section{Coupling of miniaturised gas chromatographs with mass spectrometers}

Coupling of mass spectrometers with mobile gas chromatographs long time was not possible because mass spectrometers were too complex, too large, too heavy, as well as too power-consuming to be used as mobile devices. However, thanks to the developments of size reduction technology and new methodological solutions over the last twenty years, several types of miniature mass spectrometers (MMSs), understood as portable (field deployable) or handheld devices have been proposed [4-6].

An important solution for reducing the size of spectrometers are sample injection port systems, in which ions can be produced under ambient as well as in the open-air conditions without prior sample preparation [7]. It has been shown that compatible with MMSs are different ambient ionization methods, including low-temperature plasma, paper spray, and extraction spray [8].

Although reducing the size of mass spectrometers can lead to poorer performance of the device compared to laboratory spectrometers, the mass spectrometers thumbnails are designed to achieve the right resolution, low limit of detection, satisfactory accuracy and, above all, the possibility of automatic operation. In many chip-based element designs, in addition to other benefits, the operations performed during the analysis were simplified. 
It was possible in result of, among other things, introducing mild ionization techniques, such as electrospray ionization (ESI) and matrix-assisted laser desorption ionization (MALDI) that allow the conversion of biomolecules into ions. ESI can efficiently be interfaced with separation techniques enhancing their roles in the life and health sciences. MALDI has the advantage of producing singly charged ions of peptides and proteins, minimizing spectral complexity.

Regardless of the ionization source, the sensitivity of a mass spectrometer is related to the mass analyser where ion separation occurs. Both quadrupole (cylindrical or linear) and time of flight (TOF) mass analysers are commonly used, and they can be configured together as Q-TOF tandem mass spectrometric instruments. Tandem mass spectrometry (MS/MS) is the result of coupling two or more mass analysers. Coupling of a quadrupole and TOF instruments resulted in the production of high-resolution mass spectrometers. Innovative toroidal ion traps technology were also useful in MS miniaturisation.

Ambient mass spectrometry (AMS) has become increasingly important in the last 15 years thanks to the ability to analyse multiple substances at the level of traces in complex matrices, with little pre-preparation of the sample or even without preparation [9]. AMS allows for fast in-situ analysis and in real time, usually combined with high resolution mass spectrometry. Among AMS techniques, paper spray ionization (PSI) is particularly popular thanks to its simplicity, versatility, sensitivity, and cost-effectiveness. Since its introduction in 2010, PSI has been successfully used to analyse pharmaceuticals and metabolites in biological samples, forensics, and food analysis.

Among the mass analysers in MMS design, the most interesting are the ion trap analysers (quadrupole ion trap or Paul trap, transform ion cyclotron and newly developed orbitrap). They are much smaller than beam-type mass analysers, such as TOF and sector mass analysers. In addition, ion trap analysers can be used to perform multistage MS/MS in a single device. One of the advantages of the new traps (e.g. toroidal ion trap) is the ability to operate at higher pressures than beam-type analysers $[10,11]$. Recent developments of miniature ion trap mass spectrometers were reviewed in 2018 [12].

The vacuum system, which includes a vacuum pump and a vacuum manifold with its interfaces, is usually the largest component of the mass spectrometer and consumes the most electricity. MMS uses miniature-turbo pump configurations like lab-scale instruments. The latest pumps weigh just $500 \mathrm{~g}$ and consume 18 Watts of power, providing a pressure of $10^{-8}$ torr [13].

There is a series of miniaturised mass spectrometers based on quadrupole ion trap called Mini-10, Mini-11, and Mini-12 [14]. Mini MS 10 weighs $10 \mathrm{~kg}$ with electrospray ionization and paper spray ionization. The $8.5 \mathrm{~kg}$ Mini-11 and $25 \mathrm{~kg}$ Mini-12 can provide resolution mass spectra up to $600 \mathrm{~m} / \mathrm{z}$. Mini-12 can analyse directly complex samples without sample preparation or, to some extent, without chromatographic separation. The latest model mini 2000 is an upgraded miniature $(13 \mathrm{~kg}$ ) mass spectrometer with continuous atmospheric pressure interface and a more powerful pumping system. This model has the capability to perform tandem mass spectrometry [14].

The MMS 1000 was originally designed for NASA for monitoring air quality on the International Space Station. This model has a cylindrical ion-trap MS with MS/MS capability. MMS 1000 allows for compounds to be analysed in a wide range of molecular masses (35-450 Da) with a resolution less than 0.5 Da. The other two miniature spectrometers are handheld M908 (2 kg) with solid, liquid, and gas multi-phase detector, and spectrometer 3500 and 4000 using single quadrupole mass analyser [15]. There 
are several other instruments fabricated using ion trap mass analysers such as Tridion- 9 and Chemsense 600. An extensive review on miniaturisation in MS has been published recently [16].

Presently, mobile gas chromatographs coupled with mass spectrometers have been proposed by some companies, such as: Griffin G-510 (FLIR Detection), Torion T-9 (Perkin Elmer), Hapsite ER (Inficon AG).

\section{Coupling of mobile gas chromatography with ion mobility spectrometers}

Ion mobility spectrometry (IMS) is an analytical technique involving the use of ion movement in the gas phase (drift gas) in the electric field [19]. Qualitative analysis in IMS is based on the differences in mobility of ions formed by ionization of molecules of the analysed substances. The differences in mobility are related with their weight, electric charge and shape [20]. In practice IMS is realized in three basic techniques: drift tube, field asymmetric and aspiration.

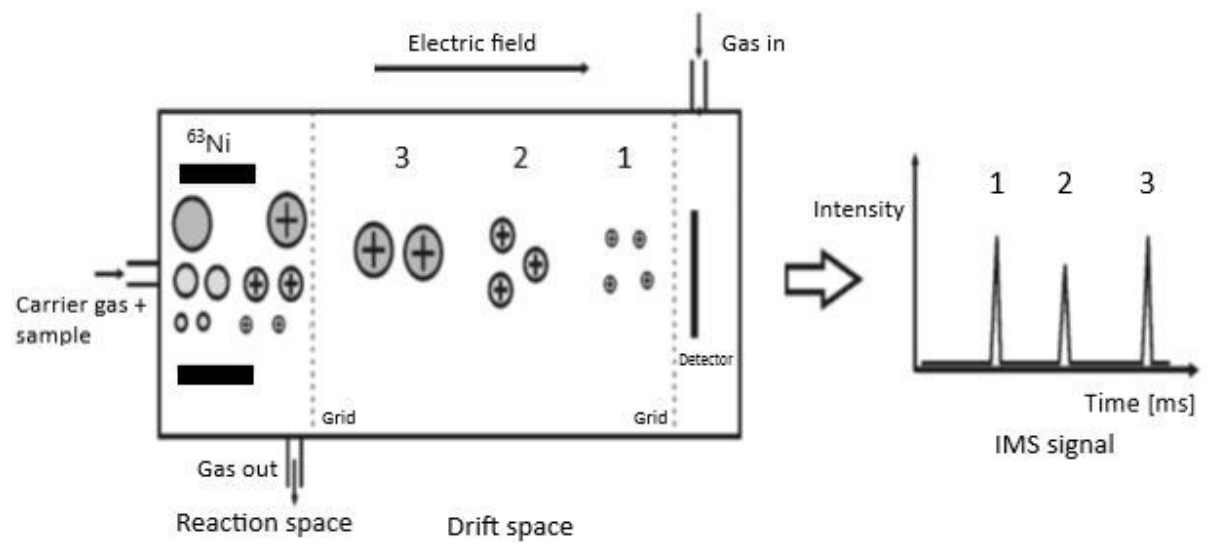

Fig. 1. Principle of operation of Drift Tube Ion Mobility Spectrometer (DT IMS)

In conventional drift tube IMS (DT IMS) ions are created in the reaction section and are separated in drift section (Fig. 1). Ionization is typically carried out using $\beta$ radiation, but other ionization procedures (photoionization, corona discharge or laser beam) can be used [21]. Ions in drift section are moving in electric field, in gas moving in opposite direction to the movement of ions, to collecting electrode. Signals are obtained in the form of peaks, the drift time of which is characterized by qualitative substances and their size quantitatively [17]. Spectrometers using this ion separation technique are most frequently used in portable and transportable instruments. DT IMS has several advantages over MS. It can distinguish between isomers which is not possible with MS. Due to the separation of individual ions, not only based on $\mathrm{m} / \mathrm{z}$ ratio but also on their active cross-section, the problem of their distinction in mass spectrum decreases. DT IMS can be easily combined with time-of-flight (TOF-MS) instruments, obtaining combined information in the form of drift time and mass spectrum [18, 22].

In 2019 a light and an easy-to-manufacture miniaturised drift tube ion mobility spectrometer with $3 \mathrm{H}$ ionization source or a non-linear X-ray ionization source with $3 \mathrm{kV}$ acceleration voltage was presented [23]. Since the drift electrodes are connected to 
a dual-in-line package footprint, the drift tube is easy to connect to the driver electronics (plug and play).

In field asymmetric ion mobility spectrometry (FAIMS) (Fig. 2), frequently referred to as differential mobility spectrometry (DMS), ions are produced in the reaction section and transferred in the carrier gas stream to a separator made of two parallel plates [24]. These plates are electrodes used to produce a perpendicular variable electric field. This field is produced by attaching separation voltage to the electrodes of a radio frequency. Ions transferred in the carrier gas, e.g. filtered air, oscillate between electrodes and become neutralized on them. The strong asymmetry of the electric field in the separator makes the ion mobility value different for both directions of oscillating motion. Adding a constant component to the variable separation voltage in the form of compensating voltage allows the ion to pass with a specified mobility throughout the separator. It is neutralized at the end of the separator on the bulk electrode. The identification of ions is based on the compensating voltage value, which allows them to pass through the entire separator. The output signal of the DMS spectrometer is the relationship of the current of the aggregate electrode to the compensating voltage. The advantage of DMS are: simplicity, compact design and the ability to simultaneously record negative and positive ions at potentially high resolution. DMS can be easily miniaturised using micro-electro-mechanical systems (MEMS) [16].

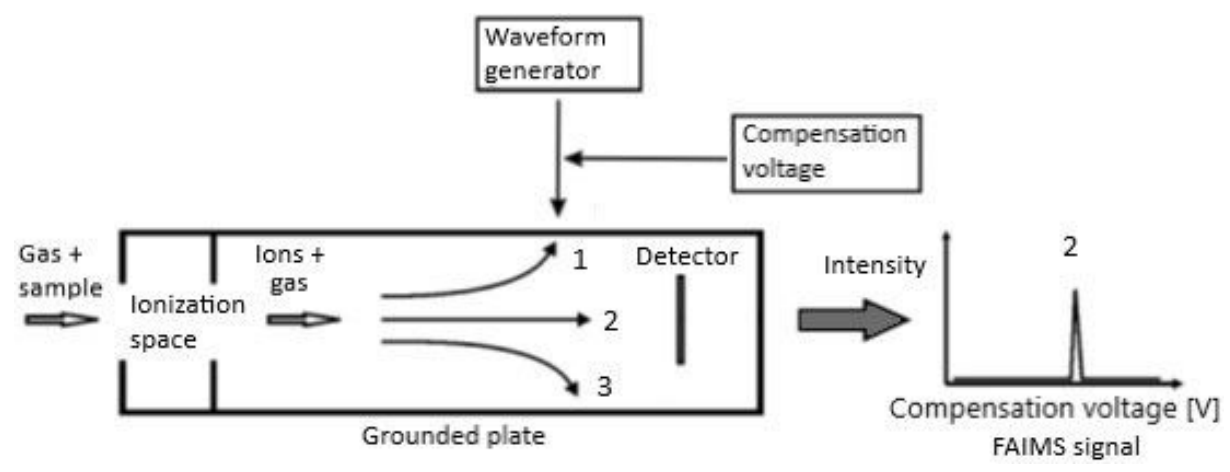

Fig. 2. The operating principle of Field Asymmetric Ion Mobility Spectrometer (FAIMS)

Ion mobility spectrometers enable getting very good results but their separation performance is not good, because the same analyte may form different peaks in result of forming dimer ions [25]. Therefore, the coupling of IMS spectrometers with gas chromatographs was obvious solution to escape this problem [26]. This combination allow getting two dimensional information, consisting retention time, drift time or compensation voltage $[18,22]$. Presently, the following instruments are commercially available: EGIS Defender, Smith Detection GC-IONScan.

\section{Selected examples of application of mobile GC-MS}

At the beginning the mobile GC-MS instruments were used in practice in NASA spacecrafts in 1970s. They were used in Pioneer and Viking projects for exploring Venus atmosphere [27, 28]. A prototype of gas chromatograph with a time-of flight type mass spectrometer (TOF-MS) for in-site measurement on the Moon was tested in 2015 [29]. 
Different types of the mobile GC-MS systems were developed for the analysis of chemical compounds on the Earth, for in-site applications, e.g. for determination of air, water and soil pollutants [30]. A continuous unattended operation of portable GC-MS system for 12 or 24 hour for monitoring applications in the field has been shown.

Environmental and forensic applications of field-portable GC were overviewed in 2001 [31]. Portable GC-MS systems have been used within a variety of different industries, including their extensive use for analysis of hazardous pollutants, e.g. for determination of pesticides residues in water, using sequential stir bar sorptive extraction-thermal desorption (SBSE) [32].

GC-MS devices used for analysis of typical environmental pollutions may be also applied for determination of toxic materials used for military purposes [11, 33, 34]. The difference relies on using appropriate chromatographic columns and data bases of mass spectra of investigated analytes. Mobile GC-MS devices are used for analysis of CWA on the battlefield or after terroristic attacks [20]. These system were deployed to conventional military forces for use in theatre to detect and identify toxic compounds, including CWAs. Such instruments are deployed in field laboratories and in battle vehicles. The explosives present in water have been analysed [35].

A portable GC-MS system was capable to distinguish between undamaged and mechanically damaged plant treatment, and plant environments [36]. The system utilising needle trap adsorbent technology to collect and analyse in situ, enable to detect 31 volatiles. Each GC-MS run was completed in less than 3 min.

\section{Selected examples of application of mobile GC-IMS}

Coupling of mobile GC-DT IMS has been applied, like GC-MS systems, in space exploration [27, 28]. In 2001, the Volatile Organic Analyser was placed on International Space Station (ISS) for monitoring of spacecraft cabin-air quality. The device has been used for analysis of 21 compounds on ISS up to 2009 [37]. In that year this analyser was replaced by the Air Quality Monitor (AQM), in which GC was coupled with DMS spectrometer [38, 39]. Such instruments, equipped with two different columns, enable analysis of compounds with different polarities.

In terrestrial applications GC-IMS devices are used for analyses of air pollutants, drugs, explosives [40, 41] and CWA [42, 43]. In food industry it was applied for rapid assessment of egg products freshness [44]. In medicine, six compounds present in exhaled air supposed as respiratory markers of different diseases have been found [26]. A selection of chemical marker compounds was performed using solid phase microextraction gas chromatography (SPME-GC) technique and associated to the parallel IMS volatile fingerprinting.

Application of fast chromatography enable performing analyses in short time. Some IMS devices may work as self-reliant, when chromatographic separation is not needed. The liquid or solid samples in some GC-IMS instruments were injected using head space solid phase microextraction (SPME) or needle trap attachments [45].

\section{Conclusion}

Miniaturization of conventional gas chromatographs and mass and ion mobility spectrometers has been possible using innovative technologies (MEMS, NEMS) and a new approaches (microfluidics). In the result significant advancements have been made in recent 
three decades, leading to availability of several portable instruments providing better portability, in situ and fast analysis, robustness and affordability. The construction of contemporary hybrid devices, comprising gas chromatographs and mass or ion mobility spectrometers may be fully mobile, handheld and transportable. Mobile GC-MS instruments have an advantage over GC-IMS devices in terms of selectivity, but LOD of the latter is much higher. The advantage of GC-IMS is that they are largely portable while GC-MS are mainly transportable that weighs up to $20 \mathrm{~kg}$. Most GC-MS systems are transportable, but some GC-MS are also handheld devices. GC-IMS devices operate under atmospheric pressure without vacuum generation systems. The range of applications of mobile GC-MS and GC-IMS instruments is very broad and similar to a conventional laboratory instruments. Especially important is their applications in planetary space cosmic research and for the analyses of environmental pollutants, including as specific as CWAs.

\section{Acknowledgements}

The authors dedicate this article in the memory of Professor Witold Wacławek, an outstanding scientist and creative defender of the environment, the founder of an annual ecological conference: Central European Conference ECOpole.

\section{References}

[1] Gałuszka A, Migaszewski ZM, Namieśnik J. Moving your laboratory to the field - advantages and limitations of the use of field-portable instruments in environmental sample analysis. Environ Res. 2015;140:593-603. DOI: 10.1016/j.envres.2015.05.017.

[2] Witkiewicz Z, Wardencki W. Transportable, portable and micro gas chromatographs. Anal Chem: Indian J. 2019;19:1-12. DOI:10.37532/0974-7419.2019.19(1).142.

[3] Qu H, Duan X. Recent advances in micro detectors for micro gas chromatography. Sci China Mater. 2019;62(5):611-23. DOI: 10.1007/s40843-018-9389-0.

[4] Makas AL, Troshkov ML. Field gas chromatography-mass spectrometry for fast analysis. J Chrom B. 2004;800:55-61. DOI: 10.1016/j.jasms.2008.06.0.

[5] Li L, Chen T, Ren Y, Hendricks PI, Cooks RG, Quyang Z. Mini 12, miniature mass spectrometer for clinical and other applications - Introduction and characterization. Anal Chem. 2014;86:2909-16. DOI: 10.1021/ac403766c.

[6] Snyder D, Pulliam C, Quyang Z, Cooks R. Miniature and fieldable mass spectrometers. Anal Chem. 2016;88(1):2-29. DOI: 10.1021/acs.analchem.5b03070.

[7] Sanders NL, Kothari S, Huang G, Salazar G, Cooks RG, Detection of explosives as negative ions directly from surfaces using a miniature mass spectrometer. Anal Chem. 2010; 82(12):5313-6. DOI: 10.1021/ac1008157.

[8] McBride EM, Mach PM, Dhummakupt ES, Dowling S, Carmay DO, Demond PS, et al. Paper spray ionization: Applications and perspectives. Trends Anal Chem. 2019;118:722-30. DOI: 10.1016/j.trac.2019.06.028.

[9] Xiao Y, Deng J, Yao Y, Fang L, Yang Y, Luan T. Recent advances of ambient mass spectrometry imaging for biological tissues: A review. Anal Chim Acta. 2020;1117:74-88. DOI: 10.1016/j.aca.2020.01.052.

[10] Lammert SA, Rockwood AA, Wang M, Lee M, Lee ED, Tolley SE, et al. Miniature toroidal frequency ion trap mass analyzer. J. Am Soc Mass Spectrom. 2006;17:916-22. DOI: 10.1016/j.jasms.2006.02.009.

[11] Contreras JA, Murray JA, Tolley SE, Oliphant JL, Tolley HD, Lammert SA, et al. Hand-portable gas chromatograph-toroidal ion trap mass spectrometer for detection of hazardous compounds. J Am Soc Mass Spectrom. 2008;19:1425-34. DOI: 10.1016/j.jasms.2008.06.022.

[12] Guo Q, Gao L, Zhai Y, Xu W. Recent developments of miniature ion trap mass spectrometers. Chin Chem. Letters. 2018;29:1578-84. DOI: 10.1016/j.cclet.2017.12.009.

[13] Quyang Z, Cooks RG. Miniature mass spectrometer. Ann Rev Anal Chem. 2009;2:187-214. DOI: 10.1146/annurev-anchem-060908-155229.

[14] Meng X, Zhang X, Zhai Y, Xu W. Mini 2000: a robust miniature mass spectrometer with continuous atmospheric pressure interface. Instruments. 2018;2. DOI: 2,210.3390/instruments2010002. 
[15] Hamilton SE, Mattrey F, Bu X, Murray D, McCullough B, Welch CJ. Use of miniature mass spectrometer to support pharmaceutical process chemistry. Org Process Res Develop. 2014;18:103-8. DOI: 1021/op400253x.

[16] Mielczarek P, Silbering J, Smoluch M. Miniaturization in mass spectrometry. Mass Spectrom Rev. 2020;39(5-6):453-70. DOI: 10.1002/mas.21614.

[17] Cumeras R, Figueras E, Davis CE, Baumbach JI, Gracia J. Review on ion mobility spectrometry. Part 1: Current instrumentation. Analyst. 2015;140:1376-90. DOI: 10.1039/c4an01100g.

[18] Cumeras R, Figueras E, Davis CE, Baumbach JI, Gracia J. Review on ion mobility spectrometry. Part 2: Hyphenated methods and effects of experimental parameters. Analyst. 2015;140:1391-410. DOI: 10.1039/c4an01100g. Analyst. 2015; 140:1391-1410. DOI: 10.1039/c4an01101e.

[19] Eiceman GA, Karpas Z, Hill HH Jr. Ion Mobility Spectrometry. 3rd ed. Boca Raton: Taylor Francis; 2013. ISBN: 9781439859971.

[20] Puton J, Namieśnik J. Ion mobility spectrometry. Trends Anal Chem. 2016;85:10-20. DOI: 10.1016/j.trac.2016.06.002.

[21] Satoh T, Kishi TS, Nagashima H, Tachikawa M, Kanamori-Kataoka M, Nakagawa T, et al. Ion mobility spectrometric analysis of vapours chemical warfare agents by the instrument with corona discharge ionization ammonia dopant ambient temperature operation. Anal Chim Acta. 2015;865:39-52. DOI: 10.1016/j.aca.2015.02.004.

[22] Kanu AB, Hill HH Jr. Ion mobility for gas chromatography. J Chromatogr A. 2008;1177;12-27. DOI: 10.1016/j.chroma.2007.10.110.

[23] Ahrens A, Hitzemann, Zimmermann S. Miniaturized high-performance drift-tube ion mobility spectrometer. J Ion Mobil Spectrom. 2019;22:77-83. DOI: 10.1007/s12127-019-00248-w.

[24] Schneider BB, Nazarov EG, Londry F, Vouros FP, Covey TR. Differential mobility spectrometry/mass spectrometry, history, theory, design optimization, simulations, and applications. Mass Spectrom Rev. 2015;34:687-737. DOI: 10.1002/mas.21453.

[25] Cohen MJ, Karasek FW. Plasma chromatography - a new dimension for gas chromatography and mass spectrometry. J Chromatogr Sci. 1970;8(6):330-7. DOI: 10.1093/chromsci/8.6.330.

[26] Aguilera-Herradora E, Cardenasa S, Ruzsanyi V, Sielemann S, Varcalcel M. Evaluation of a new miniaturized ion mobility spectrometer and its coupling to fast chromatography multi-capillary columns. J Chromatogr A. 2008;1214:143-50. DOI: 10.1016/j.chroma.2008.10.050.

[27] Palmer PT, Limero TF. J Am Soc Mass Spectrom. 2001;12:656-76. DOI: 10.1016/S1044-0305(01)00249-5.

[28] Grabka M, Żukowski P, Witkiewicz Z. Zastosowanie chromatografii gazowej w pozaziemskich misjach badawczych (Application of gas chromatography in extraterrestrial research missions). Aparat Bad Dydakt. 2012;17:69-77.

[29] Hofer L, Wurz P, Buch A, Cabane M, Cool P, Coscia D, et al. Planet Space Sci. 2015;111:126-33. DOI: 10.1016/j.pss.2015.03.027.

[30] Gorder KA, Dettenmaier ME. Groundwat Monit Remed. 2011;31:113-9. DOI: 10.1111/j.1745-6592.2011.01357.x.

[31] Eckenrode BA. Environmental and forensic application of field-portable GC-MS: An overview. J Am Soc Mass Spectrom. 2001;12(6):683-93. DOI: 10.1016/S1044-0305(01)00251-3.

[32] Ochiai N, Sasamoto K. Screening of pesticide residues in water by sequential stir bar sorptive extraction-thermal desorption with GC/MSD. Appl Note Agilent Technol. 2010.

[33] Leary PE, Kammrath BW, Lattman KJ, Beals GL. Deploying portable gas chromatography-mass spectrometry to military users for the identification of toxic chemical agents in theatre. Appl Spectrosc. 2019;73:841-58. DOI: 10.1177/0003702819849499.

[34] Sekiguchi H, Matsushita K, Yamashiro S, Sano Y, Seto Y, Okuda T, et al. On-site determination of nerve and mustard gases using a field-portable gas chromatograph-mass spectrometer. Forensic Toxic. 2006;24:17-22. DOI: 10.1007/s11419-006-0004-4.

[35] Bednar AJ, Russell AL, Hayes CA, Jones WT, Tackett Splichal DE, Georgian T, et al. Chemosphere. 2012;87:894-901. DOI: 10.1016/j.chemosphere.2012.01.042.

[36] Beck J, Porter N, Cook D, Gee WS, Griffith CM, Rands AD, et al. In-field volatile analysis employing a hand-held portable gc-ms: emission profiles differentiate damaged and undamaged yellow starthistle flower heads. Phytochem Anal. 2015;26:395-403. DOI: 10.1002/pca.2573.

[37] Limero T, Cheng P, Reese E, Trowbridge J. Results of the air quality monitor's experiment to measure volatile organic compounds aboard the International Space Station. 40th Int Conf Environmental Systems, Barcelona, September 2010. URI: hdl.handle.net/2346/72986.

[38] Limero T, Wallace W, James JT. Operational validation of the air quality monitor on the International Space Station. 44th Int Conf Environmental Systems, Tucson. July 2014. URI: hdl.handle.net/2346/72986. 
[39] Limero T, Nazarov EG, Menlyadiev M, Eiceman GA. Analyst. 2015;140:922-30. DOI: 10.1039/C4AN01800A.

[40] Caygill JS, Davis F, Higson SP. Current trends in explosive detection techniques. Talanta. 2012;88:80-8. DOI: 10.1016/j.talanta.2011.11.043.

[41] Cook GW, LaPuma PT, Hook GL, Eckenrode BA. J Forensic Sci. 2010;55:1582-91. DOI: 10.1111/j.1556-4029.2010.01522.x.

[42] Kwan C, Snyder AP, Erickson RP, Maswadeh PA, Ayhan B, Jensen JL, et al. IEEE Sensors J. 2010;10:451-60.

[43] Erickson RP, Tripathi A, Maswadeh WM, Snyder AP, Smith PA. Closed tube introduction for gas chromatography-ion mobility spectrometry analysis of water contaminated with a chemical warfare agent surrogate compound. Anal Chim Acta. 2006;556:455-61. DOI: 10.1016/j.aca.2005.09.031.

[44] Cavanna D, Zanardi S, Dall'Asta C, Suman M. Food Chem. 2019;15:691-6. DOI: 10.1016/j.foodchem.2018.07.204.

[45] Reyes-Garces N, Gomez-Rios GA, Souza Silwa EA, Pawliszyn J. Coupling needle-trap devices with gas chromatography-ion mobility spectrometry detection as a simple approach for on-site quantitative analysis. J Chromatogr A. 2013;1300:193-8. DOI: 10.1016/j.chroma.2013.05.042. 